\title{
Inherited electrophoretic variants detected in a Japanese population with two-dimensional gels of erythrocyte lysates
}

\author{
By N. TAKAHASHI,* J. V. NEEL, $†$ Y. NAGAHATA-SHIMOICHI,* J. ASAKAWA,* \\ Y. TANAKA* AND C. SATOH* \\ * Division of Biochemical Genetics, Department of Genetics, Radiation Effects Research \\ Foundation, Hiroshima 732, Japan \\ $\dagger$ Department of Human Genetics, University of Michigan Medical School, Ann Arbor,
} Michigan 48109, U.S.A.

\begin{abstract}
SUMMARY
Genetic variation has been studied in erythrocyte lysates from 100 Japanese children and their parents by means of two-dimensional polyacrylamide gel electrophoresis. Fifty-five polypeptides selected without respect to variability were considered suitable for scoring. Genetic variation was encountered in 14 of these polypeptides. Family data show that the segregation of 13 variants is consistent with an autosomal codominant mode of inheritance; the remaining variant exhibits a sex-linked mode of inheritance. Of 8 presumably identical polypeptides found variable in Japanese and/or Caucasians, differences in the occurrence or allele frequencies of polymorphisms were recognized for four. Contrary to the experience of some investigators, the amount of variation and the ethnic differences we are encountering indicate that two-dimensional polyacrylamide gel electrophoresis is a sensitive tool for the study of genetic events.
\end{abstract}

\section{INTRODUCTION}

In a series of recent publications (Asakawa et al. 1984, 1985; Neel et al. 1984 ; Rosenblum et al. $1983 a, b, 1984$ ), we have been exploring the usefulness of the two-dimensional polyacrylamide gel (2-D PAGE) technique in the study of genetic variation, with especial reference to adapting this technology to the study of mutation. In the course of this series, genetic variation has been studied in the polypeptides of plasma samples from 3 kinds of populations (Caucasoids, Amerindians and Japanese) (Asakawa et al. 1985). Interethnic comparisons of the index of heterozygosity for the 11 polypeptides which could be scored in all three populations and which can be regarded as randomly selected with reference to genetic variability revealed the following: Caucasoids, 8.0 $\pm 1 \cdot 1 \%$; Japanese, $5 \cdot 7 \pm 0 \cdot 7 \%$; and Amerindians, $4.5 \pm 0.6 \%$. In addition to the emerging differences in heterozygosity indices, ethnic differences in allele frequencies appeared to characterize several of the variable polypeptides.

The present communication will report on the variation encountered in preparations of erythrocyte lysates from a Japanese population, compare these findings with the results of previous similar studies on a predominantly Caucasian population, and describe a battery of polypeptides suitable for studies of spontaneous and induced mutation rates. The findings in this study will in general confirm the results on Caucasoids regarding the levels of heterozygosity in erythrocyte lysate proteins but, as with respect to the plasma proteins, ethnic differences appear to be emerging for several polypeptides. 

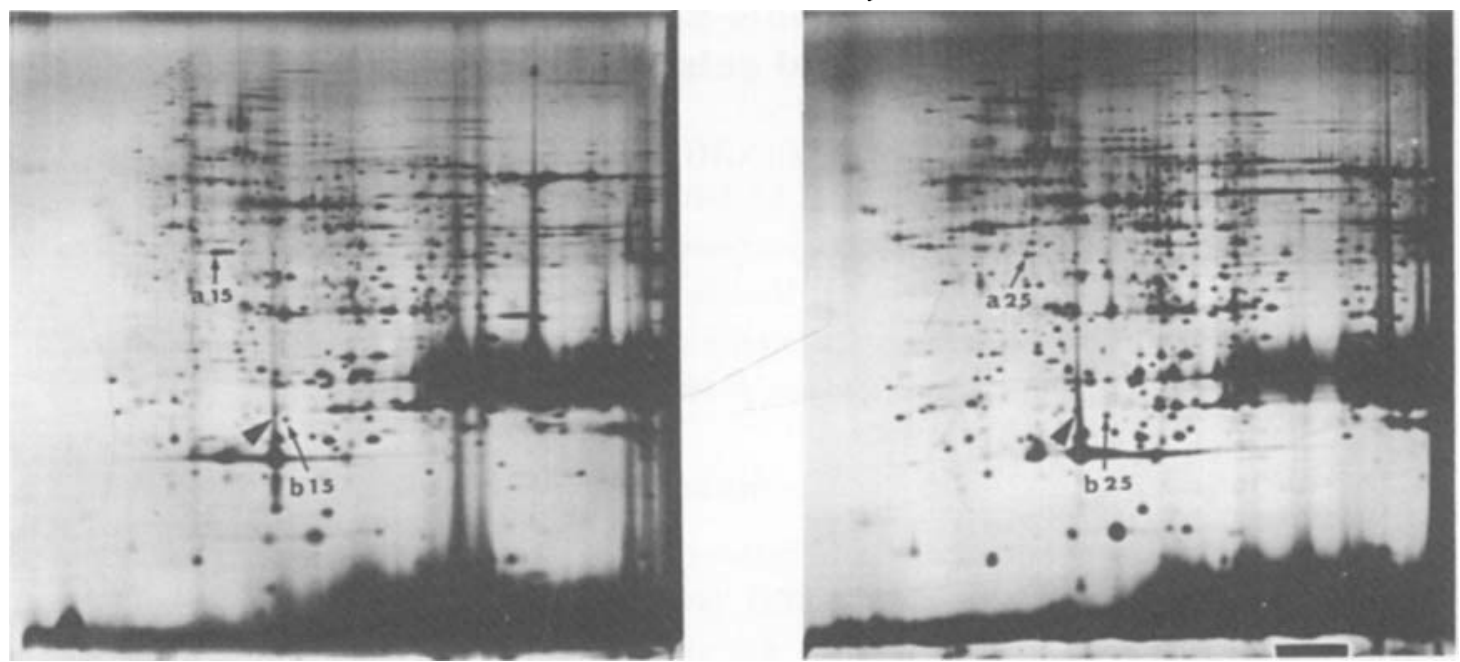

Fig. 1. Effect of $10^{\circ} \mathrm{C}$ difference in the temperature of first-dimension isoelectric focusing on polypeptide migration in $2-\mathrm{D}$ gels. The first-dimension of gel $\mathrm{A}$ was run at $15^{\circ} \mathrm{C}$ and of gel $\mathrm{B}$ at $25^{\circ} \mathrm{C}$. The positions and appearance of numerous polypeptides differ in the two gels, as illustrated by polypeptides C-007 and D-027 (see Fig. 2). C-007 consistently appears as a streak at $15^{\circ} \mathrm{C}$ (a15) but a discrete spot at $25^{\circ} \mathrm{C}$ (a25). D-027 at $15^{\circ} \mathrm{C}$ migrates $3 \mathrm{~mm}$ cathodally to the reference streak indicated by an arrow head (b15) but $7 \mathrm{~mm}$ cathodally at $25^{\circ} \mathrm{C}$ (b25).

\section{TECHNICAL CONSIDERATIONS}

Samples were stored as packed red cells in liquid nitrogen. Cells were lysed by adding 2 volumes of distilled water to 1 volume of packed red cells. Lysates were centrifuged 20 min at $50000 \mathrm{~g}$ in a Sorvall RC-5B Refrigerated Superspeed Centrifuge (Du Pont Instruments) to pellet the red cell membranes. Prior to electrophoresis, lysates were diluted with an equal volume of solubilization solution [0.8\% Ampholine ( $\mathrm{pH} \mathrm{3.5-10)}$ (LKB), 9 M urea, $4 \%$ Nonidet P40 (NP-40), $5 \%$ 2-mercaptoethanol] and kept at least 20 min at room temperature. Twodimensional electrophoresis was carried out using the Iso-Dalt system (Electro-Nucleonics, Oak Ridge, TN, USA), as described by Rosenblum et al. (1984) with four modifications: (1) A series of different mixtures of Ampholine have been tested to obtain the $\mathrm{pH}$ gradient that gave the widest and sharpest distribution of lysate protein in the first-dimension. A 3:1 ratio of $\mathrm{pH} \mathrm{3 \cdot 5-10}$ and pH 5-7 Ampholines to make a 2\% Ampholine yielded the best result under our conditions. (Only one batch number of each Ampholine was used during the course of this work.) (2) An overlay buffer, containing $0.4 \%$ Ampholine (pH 3.5-10), 2 m urea, $2 \%$ NP-40, and $2 \%$ 2-mercaptoethanol, was used for protecting the proteins of erythrocyte lysates from denaturation caused by cathode electrode solution. Following focusing, the gels were extracted from the tubes and equilibrated as described by Rosenblum et al. $(1983 a)$. Gels were stored at $-80^{\circ} \mathrm{C}$ until run in the second-dimension. Second-dimension sodium dodecyl sulphate (SDS) electrophoresis was performed as previously described (Asakawa et al. 1985). (3) The polypeptide patterns in the gels were visualized by the modified silver staining method of Wray et al. (1981). (4) Initially, the first-dimension isoelectric focusing was performed at night at ambient temperature. Since the laboratory is unheated at night, there was considerable variation in the ambient 
temperature, which fact influenced polypeptide migration (Fig. 1). During the latter half of these studies, all first-dimension gels were run in a constant-temperature room at $25^{\circ} \mathrm{C}$. Molecular weight (MW) standards used were phosphorylase A (94000), bovine serum albumin $(67000)$, ovalbumin $(43000)$, carbonic anhydrase (30000), soybean trypsin inhibitor (20100), and lysozyme (14400).

\section{SOURCE OF SAMPLES}

Elsewhere we have described an extensive effort to determine whether exposure to the radiation of the atomic bombs has increased the frequency of mutations detectable with one-dimensional electrophoresis (1-DE) and enzyme activity measurement in the children of survivors (Neel et al. 1980; Satoh et al. 1982, 1983, 1985). The samples employed in the present study had been obtained when, in the course of the program just referenced, an examination of the nuclear family was necessary to determine if either a rare electrophoretic variant or enzyme activity variant was the result of mutation in the preceding generation. The packed erythrocytes of child, father, and mother had been stored in liquid nitrogen for from 0.5 to 4.5 years. We will base our tabulations in this paper on the findings in the children but confirm the genetic nature of any putative variant by an examination of the father and mother. The full sample of children consists of 56 males and 44 females. Although some of these parents had sustained significant radiation exposures at the time of the bomb detonations, all variants encountered proved to be inherited, so that the question of a radiation effect does not enter into these data.

\section{RESULTS}

Developing the map. As the first step in developing a battery of highly reproducible polypeptides for scoring, an individual who had not previously examined these particular gels for genetic variation selected a battery of 72 spots as suitable for analysis. Our standard criteria are relative isolation of the spot and a density that would be clearly visible if the intensity of the spot were reduced to half normal. An attempt was then made to score these polypeptides for genetic variation. It developed that 17 of these spots could not be unambiguously scored with an accuracy $\geqslant 95 \%$, and they were eliminated from the battery, as unsuitable to the study of mutation.

The location of the remaining battery of 55 spots is shown in Fig. 2. A principal focus of this paper is the question of ethnic differences in lysate variants between Japanese and predominantly Caucasoids, the latter as studied in Ann Arbor, Michigan (Rosenblum et al. 1984). Although the differences in the techniques employed in the two locations were relatively small, only 27 of the 46 spots analysed in Ann Arbor could, on the basis of the constellation of spots in which they participated, be identified with the necessary high level of confidence in this study. The equivalences are (Ann Arbor designation given first) : A-002, B-002; B-007, D-012; B-009, C-009; B-011, A-003; C-002, B-005; C-005, D-002; C-006, D-006; C-007, D-008; C-008, D-011; C-009, D-015; D-007, C-011; D-009 and D-010, C-012; D-013, D-027; D-014, C-022; D-015, D-030; D-019, C-017; E-001, D-017; E-002 and E-003, D-018; E-004, D-020; E-005, D-021; E-009. D-028; E-010, D-029; E-011, D-031; E-013, D-024; F-002, E-004. 


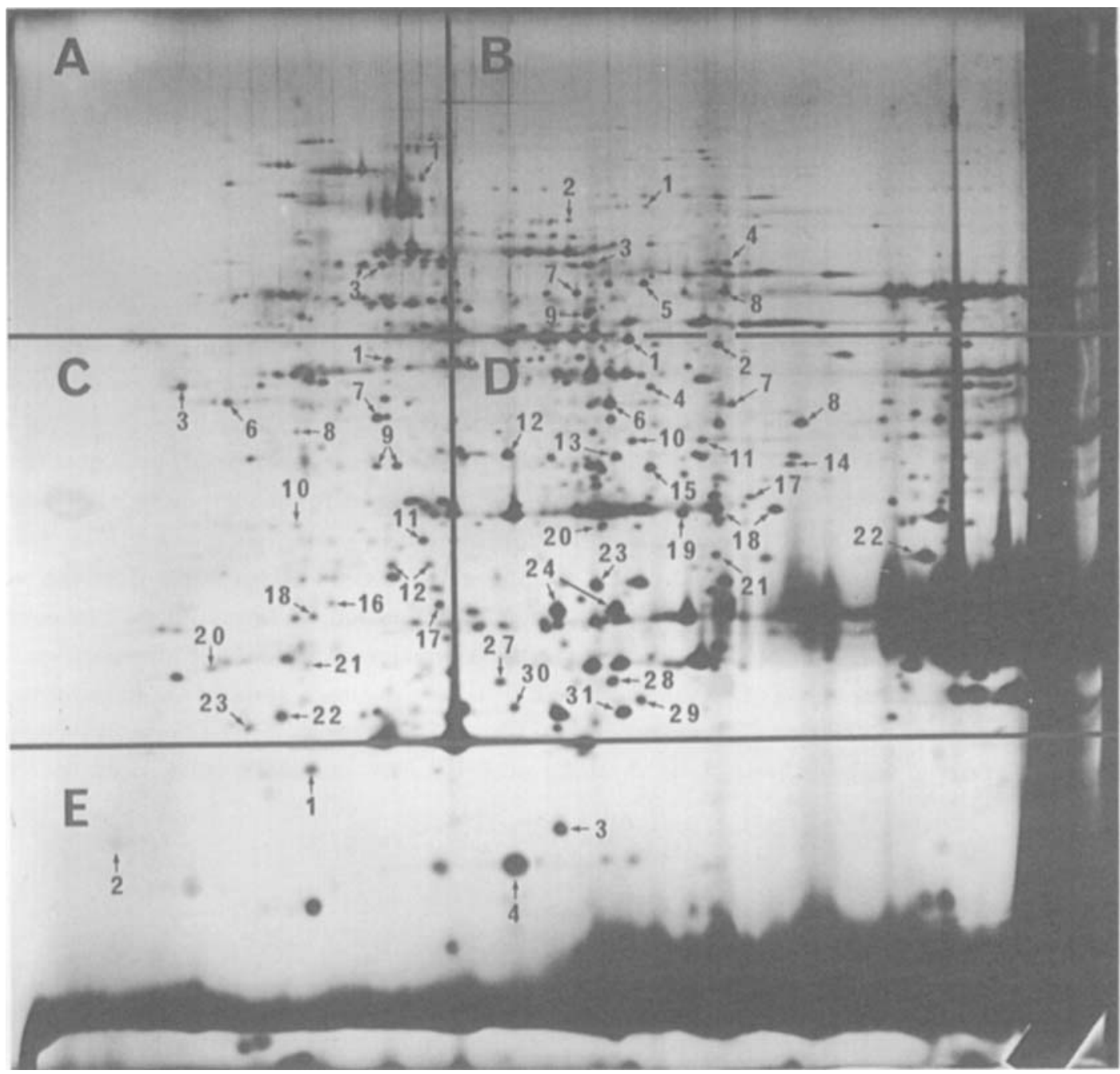

Fig. 2. Designation of the red cell lysate polypeptides scored in this study. The gel pattern has been subdivided into 5 convenient regions, and the polypeptides suitable for scoring in each region numbered consecutively. The pattern is oriented with acidic side to the left and basic side to the right.

Genetic variation. Table 1 presents the findings with reference to the 14 polypeptides exhibiting genetic variation. In general, the occurrence of a variant was accompanied by an obvious decrease in the intensity of the spot occupying the normal position. Eight of the variable polypeptides had been identified in the Ann Arbor gels; their designations and the findings are also shown in Table 1 . Of the 6 variable polypeptides in Japanese for whom no Caucasoid counterpart has been identified, none of them is found in a position which makes it likely it corresponds to the one remaining unmatched variable polypeptide in Caucasoids (C-004).

Allele frequencies are also presented in Table 1. For none of the variable polypeptides in Japanese does the distribution of phenotypes differ from Hardy-Weinberg equilibrium proportions. This includes polypeptide C-022, whose counterpart in the Ann Arbor gels, D-014, did show such a departure (supported by genetic data). The validity of all the Japanese variants 


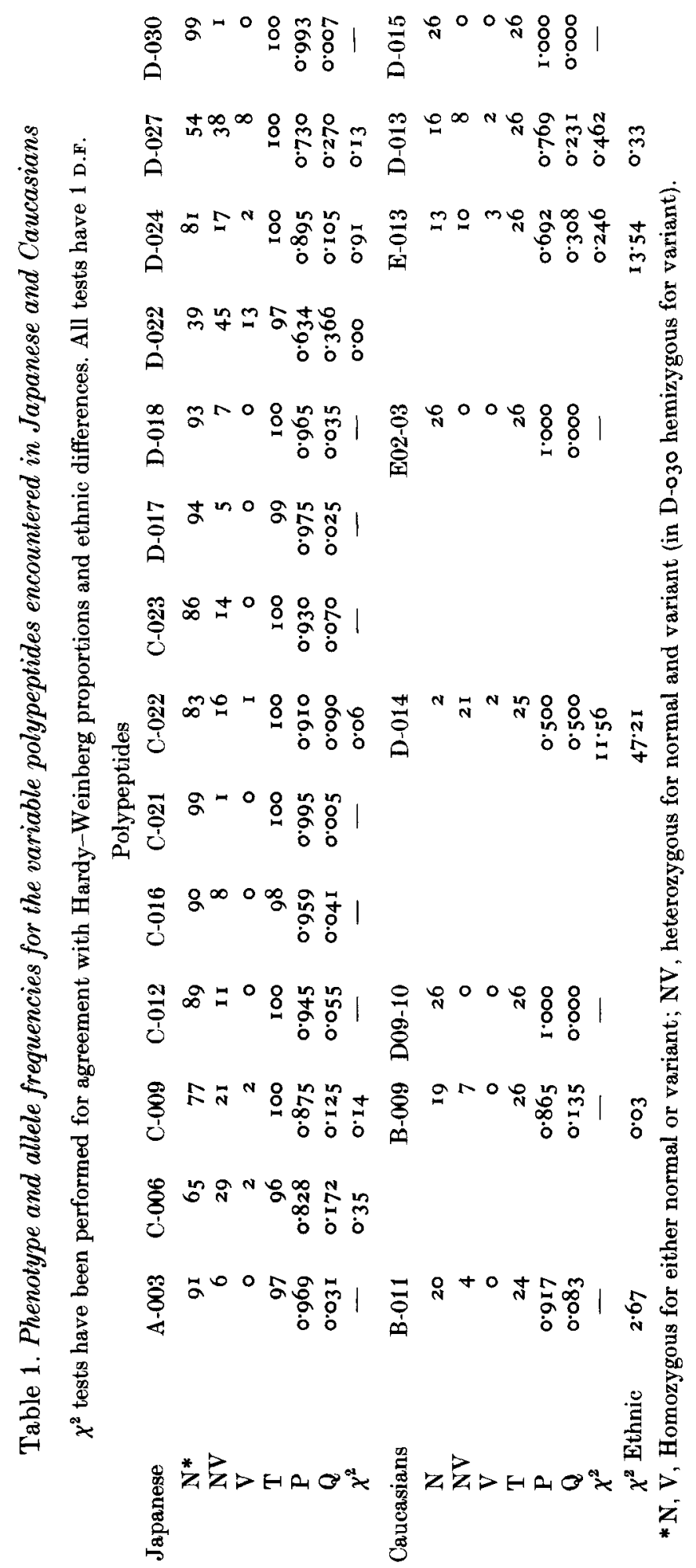


a. A 003

b. C 006

C. C 009

d. C 012

e. C 016
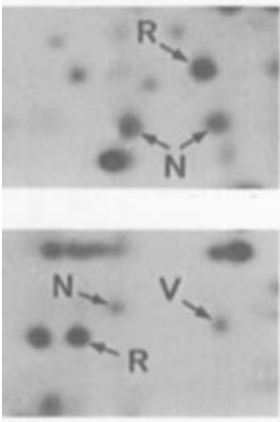

f. C 021

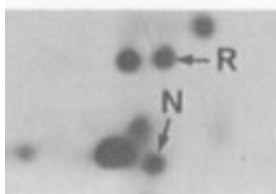

g. C 022

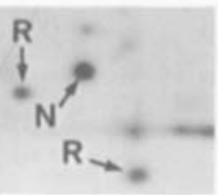

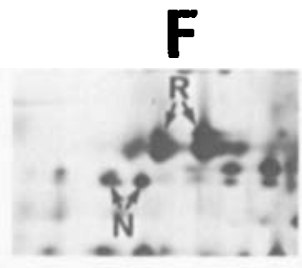
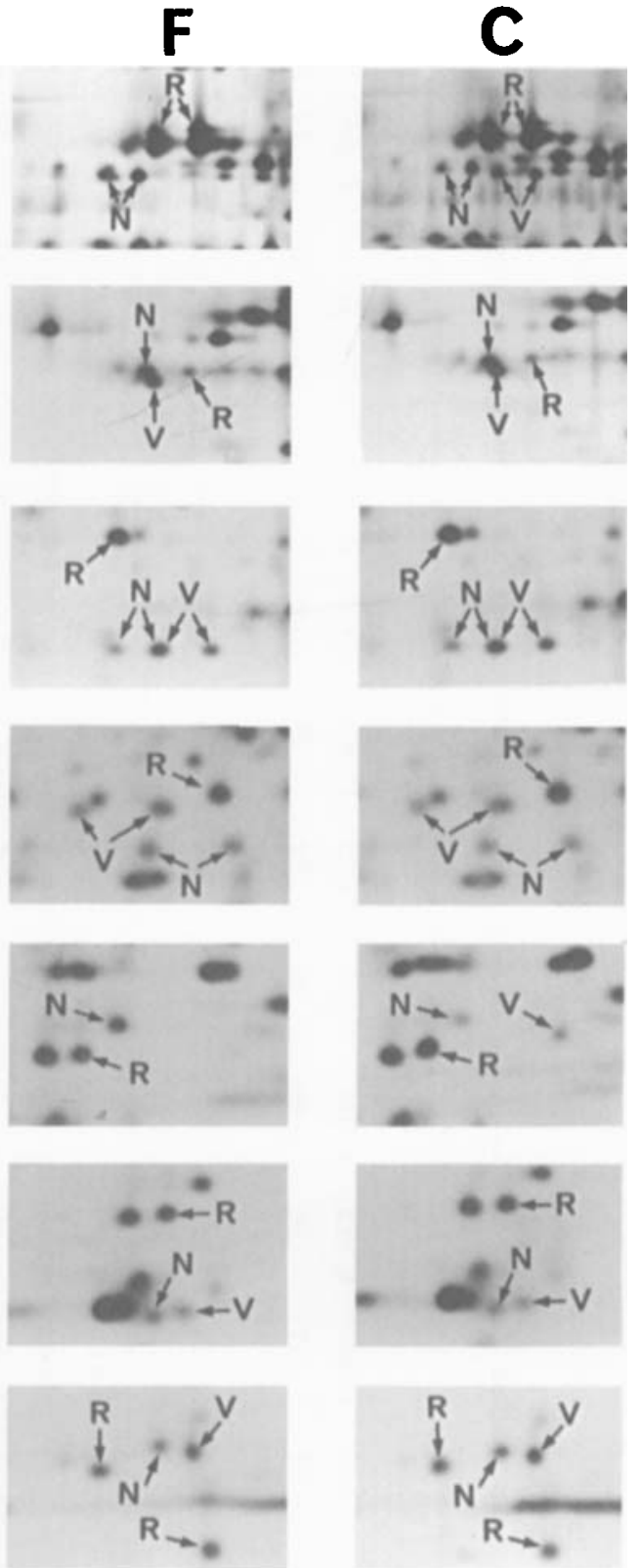

Fig. 3. Photographs of the genetic variants observed in this study. The various variants are designated as $\mathrm{V}$ and the corresponding normal polypeptides as $\mathrm{N}$. In all photos, a convenient reference spot $(\mathbf{R})$ has been designated. In row $a, c$ and $d$, both the normal and variant phenotypes are characterized by a set of two spots having almost equal staining intensity. A detailed description of the findings is in the text.

entered in Table 1 has been confirmed by the presence of the variant in the father and/or mother. Out of a possible total of 5500 spots, only 52 could not be scored $(0.9 \%)$. A total of 219 polypeptides exhibited heterozygosity for a genetic variant, yielding an index of heterozygosity of $4 \cdot 0 \pm 0 \cdot 3 \%$.

At this writing, only three of these 55 spots have been identified, C-007 as actin (ACT), C-022 
as glyoxalase 1 (GLO1), and D-022 as esterase D (ESD). The erythrocyte lysates of every individual sampled for this study have also been typed by 1-D E for variants of the following polymorphic systems: adenosine deaminase, esterase D, acid phosphatase-1, cytoplasmic glutamate-oxaloacetate transaminase, glutamate-pyruvate transaminase, phosphoglucomutase1, phosphoglucomutase-3, and 6-phosphogluconate dehydrogenase. With the exception of esterase $D$, none of the variation observed in these systems synchronizes with the variation observed in the 2-D PAGE preparations. In addition, rare electrophoretic variants of the non-polymorphic enzymes, esterase A (ESA), phosphohexose isomerase (PHI), peptidase A (PEPA), adenylate kinase (AK), lactate dehydrogenase (LDH), carbonic anhydrase-1 (CA1), and phosphoglucomutase-2 (PGM2), had been demonstrated in 20 of the individuals (ESA 2 individuals; PHI, 7; PEPA, 4; AK, 1 ; LDH, 2; CA1, 3; PGM2, 1, respectively) among the 100 examinees; no corresponding variation was seen in the 2-D PAGE preparations. (The high frequency of these variants reflects the fact that many of the mother-father-child erythrocyte lysate combinations used had been specifically collected because of the occurrence of an electrophoretic variant in the child.)

Description of variants. The variants encountered in this study can be briefly characterized as follows:

A-003. This polypeptide (MW 70000) appears to be identical with the B-011 of Rosenblum et al. (1984); the variant, encountered in six persons, appears to be the same as the previously identified polymorphism of B-011 (Fig. $3 a$ ). Approximately $3 \mathrm{~mm}$ anodally is a second spot which varies in a coordinate fashion; this coordinate variation was not reported by Rosenblum et al. (1984). The frequency of the variant allele does not differ in the two populations.

$C$-006. This polypeptide ( $\mathrm{MW} \sim 44000$ ) has the lowest isoelectric point of any of the variant polypeptides. A single type of variant was seen in thirty-one persons (Fig. $3 b$ ). It migrates slightly cathodal to and below the normal spot, sometimes not separating clearly from the normal spot. About $1 \mathrm{~mm}$ to the left of C-006 is a faint spot which appears to vary coordinately. This is one of the more frequent variants encountered in this study; its counterpart was not observed to vary in Caucasoids.

C-009. This polypeptide (MW 39000) appears to be identical with B-009 of Rosenblum et al. (1984); the single type of variant encountered in twenty-three persons appears the same as the polymorphism of B-009 in Caucasoids and has virtually the same allele frequency (Fig. $3 \mathrm{c}$ ). Approximately $3 \mathrm{~mm}$ anodally is a second spot which varies in a coordinate fashion; confusion may arise because the more anodal variant spot may be superimposed on the more cathodal normal spot.

C-012. This polypeptide (MW 33000) was observed to vary in eleven persons. In these persons a second spot $5 \mathrm{~mm}$ anodally varied coordinately (see Fig. $3 d$ ). The variant spots migrate $5 \mathrm{~mm}$ anodally and $3 \mathrm{~mm}$ above the normal. As the more cathodal spot of this variant partially overlaps with a faint spot representing an independent gene product, this variant spot appears to stain more intensely than normal in heterozygotes. The two normal spots of this system appear to correspond to spot D-009 and D-010 of Rosenblum et al. (1984); no variation was observed in this pair of polypeptides in Caucasoids.

C-016. This polypeptide (MW $\sim 31000$ ) exhibited in eight persons a variant which migrated $9 \mathrm{~mm}$ cathodally and $1 \mathrm{~mm}$ below the normal position (Fig. $3 e$ ).

C-021. This polypeptide ( $\mathrm{MW} \sim 28000$ ) was observed to vary in a single individual, who 


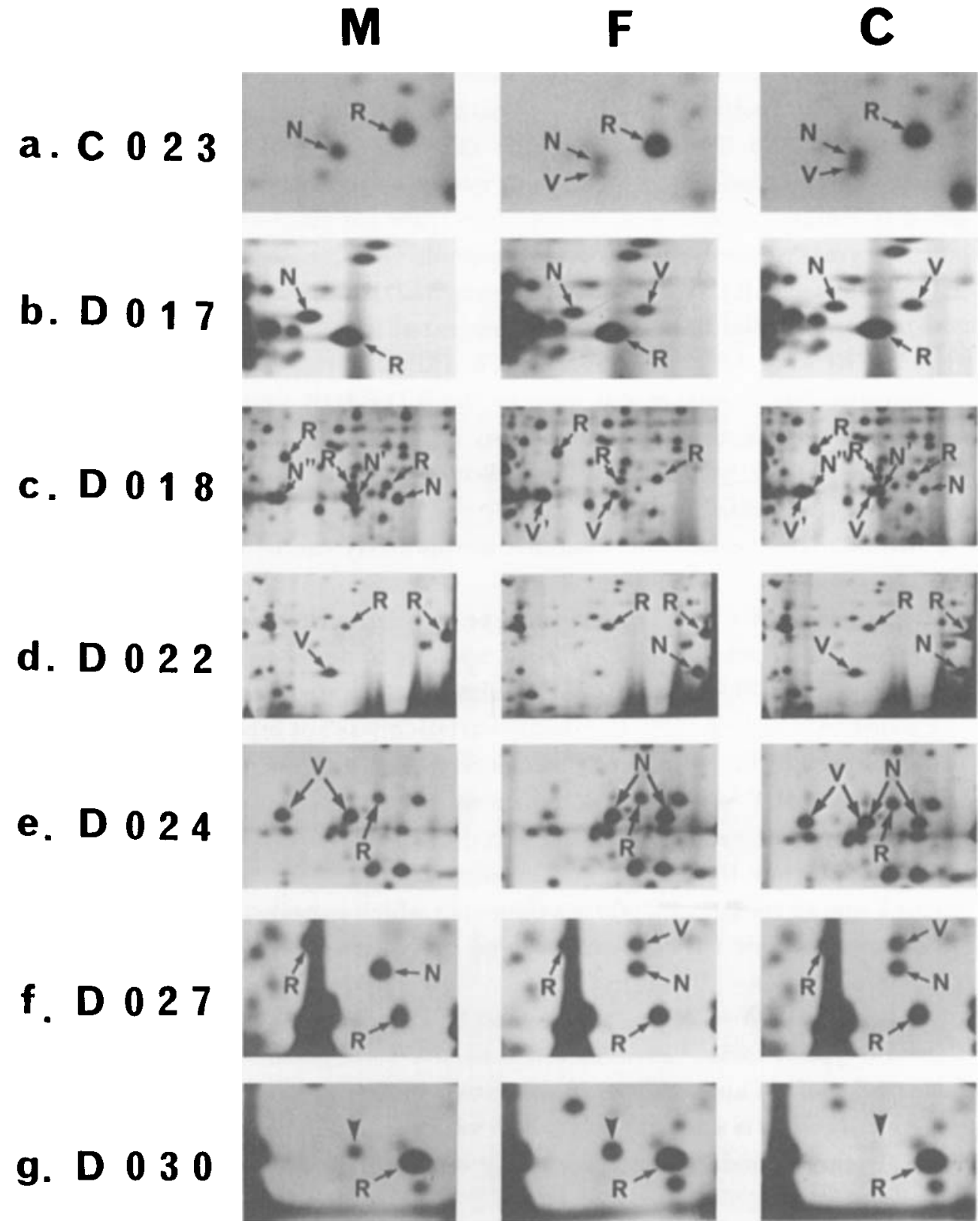

Fig. 4. Further photographs of the genetic variants observed in this study. The system of designation is the same as in Fig. 3. In rows $c$ and $e$, the phenotype is a set of two or three spots which vary concordantly. Row $g$ illustrates a sex-linked null variant. A detailed description of the findings is in the text.

exhibited a spot $2 \mathrm{~mm}$ cathodally and slightly higher in the MW axis (Fig. $3 f$ ). This spot was also present in the father and one sibling, so that the genetic nature of the spot is confirmed. The variant in heterozygotes appears of equal intensity to the normal polypeptide.

C-022. This polypeptide ( $\mathrm{MW} \sim 26000$ ) appears to be identical with D-014 of Rosenblum et al. (1984). It has recently been identified as GLO1 by Goldman et al. (1985). The single type of 
a)

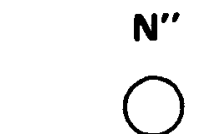

b)

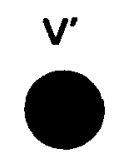

c)

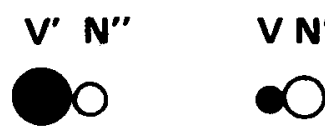

$\mathbf{N}$

O

$\mathbf{V}$

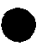

$\mathbf{V} \mathbf{N}^{\prime} \quad \mathbf{N}$

0

Fig. 5. Diagrammatic representations of genetic variation involving D-018 (a) Mother; normal homozygote. $(b)$ Father; variant homozygote. $(c)$ Child; heterozygote. A detailed description of the findings is in the text.

variant observed in seventeen persons, migrating $3 \mathrm{~mm}$ cathodally and slightly below the normal position (Fig. $3 g$ ), corresponds to the polymorphism of this polypeptide observed in Ann Arbor but the allele frequencies differ significantly $\left(\chi^{2}=47 \cdot 2\right.$, D.F. $\left.=1, P<0.001\right)$. The variant usually stains more intensely than normal in heterozygotes.

C-023. This polypeptide ( $M W \sim 25000$ ) exhibited a variant in the $\mathrm{MW}$ axis in fourteen persons (Fig. 4a). The variant is found about $1 \mathrm{~mm}$ below the normal position, and in heterozygotes is of similar staining intensity to the spot in the normal position.

D-017. This polypeptide ( $M W \sim 37000$ ) exhibited the same type of variant in five persons. The variant migrates $5 \mathrm{~mm}$ cathodally to normal and in heterozygotes appears of equal intensity to the normal spot (Fig. 4b).

$D$-018. This polypeptide (MW 36000) was observed to vary in seven persons. Figs. $4 c$ and 5 illustrate the findings in a heterozygous child whose mother was homozygous for the normal allele and father homozygous for the variant allele. In individuals with the variant, two other spots, arbitrarily designated $\mathrm{N}^{\prime}$ and $\mathrm{N}^{\prime \prime}$ according to their relative distance from the cathodal side, varied coordinately with $\mathrm{N}$. $\mathrm{N}^{\prime}$ and $\mathrm{N}^{\prime \prime}$ respectively migrate about 10 and $22 \mathrm{~mm}$ anodally to $\mathrm{D}-018$. The variant (V) of D-018 migrates $12 \mathrm{~mm}$ anodally to $\mathrm{N}$ and slightly anodally to $\mathrm{N}^{\prime}$, sometimes partially overlapping $N^{\prime}$. The second variant spot $\left(V^{\prime}\right)$ appears to overlap with a spot representing an independent gene product, so that this spot appears to stain more intensely than the second normal spot $\left(\mathrm{N}^{\prime}\right)$ in heterozygotes. A postulated third variant spot $\left(\mathrm{V}^{\prime \prime}\right)$ could not be identified; its expected position corresponds to a very congested region of the gel. Two of the normal spots ( $\mathrm{N}$ and $\mathrm{N}^{\prime}$ ) of this system appear to correspond to spots E-002 and E-003 of Rosenblum et al. (1984); no variation was observed in this pair of polypeptides in Caucasoids.

D-022. This polypeptide is esterase D, MW 33000 in 2-D PAGE gels (Kondo et al. 1984a). The single type of variant encountered consistently migrates $20 \mathrm{~mm}$ anodally to the normal position in our system (Fig. 4d) (but see Kondo et al. 1984a,b). Approximately $20 \mathrm{~mm}$ even more anodally is a second spot which is faint and varies in a coordinate fashion; the more anodal normal spot may in some gels be superimposed on the main spot of the variant. The classification of 2-D gels as heterozygous or homozygous for the variant correspond in every case to the classification with 1-D E.

$D$-024. This polypeptide (MW 30000) exhibited in nineteen persons a single type of variant 


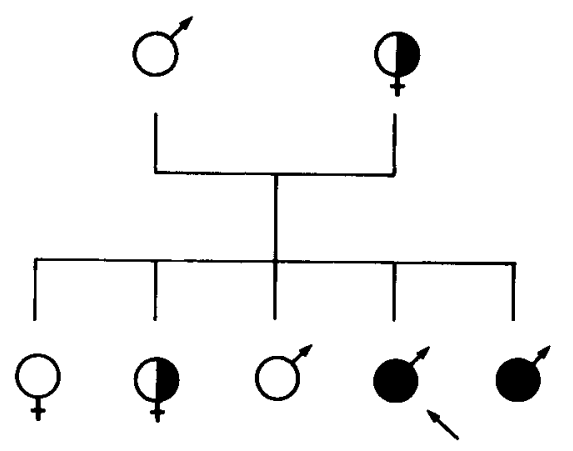

Fig. 6. Pedigree of the family illustrating segregation of a sex-linked variant of D-030 characterized by absence of gene product. $९, \sigma$; Normal female and male. $\bullet$; Hemizygous male. $\uparrow$; Heterozygous female. ; Propositus.

migrating $8 \mathrm{~mm}$ anodally to normal (Fig. $4 e$ ). A second polypeptide, $8 \mathrm{~mm}$ anodally to D-024, exhibited coordinate variation. The more cathodal of the variant spots thus corresponds to the more anodal of the two polypeptides comprising D-024. An apparently identical variant was observed in the Caucasoid series but the coordinate variation was not detected. The allele frequencies differ significantly in Japanese and Caucasoids $\left(\chi^{2}=13.54\right.$, D.F. $\left.=1, P<0.001\right)$.

$D$-027. This polypeptide (MW $\sim 27000$ ) exhibited a MW variant identical with one detected in the Ann Arbor series (D-013). The variant, present in forty-six persons, migrated about $1 \mathrm{~mm}$ above the normal position and was often of lesser intensity (Fig. 4f). The frequency of the variant allele did not differ significantly from the finding in Rosenblum et al. (1984).

$D$-030. This polypeptide ( $\mathrm{MW} \sim 26000$ ) was lacking in a single male. The mother exhibited approximately half the normal spot intensity (Fig. $4 \mathrm{~g}$ ). The study of four additional siblings exhibited the situation diagrammed in Fig. 6. We interpret these patterns as the result of sex-linkage, the mother and one sister being heterozygous for a null variant, the propositus and one brother being hemizygous for the null. This polypeptide does not seem to correspond to any of the five polypeptides whose locus was assigned to the human $\mathrm{X}$ by cell hybridization experiments (Miller et al. 1984).

In addition to these 14 polypeptides in which the case for genetic variation is clear, we have encountered in two subjects possible genetic variation involving D-008. The apparent variant pattern is complex, and a description is delayed pending further studies.

\section{DISCUSSION}

Fourteen of the 55 polypeptides scored exhibited genetic variation. Two of these 14 were variable in a single individual, so that this variation may not yet be termed polymorphic. It may, rather, correspond to the 'rare' (i.e. non-polymorphic) variants encountered with a frequency of 1-2 per 1000 in 1-DE. Conservatively then, $22 \%$ of these polypeptides were characterized by genetic polymorphisms; the corresponding figure emerging from 1-D E studies of a variety of proteins, the products of 104 different genetic loci, is $23 \%$ (Harris, 1980). For the latter, the index of heterozygosity was $6 \cdot 3 \%$. Our observed index of heterozygosity for these lysate proteins is $4 \cdot 0 \pm 0.3 \%$; the corresponding figure for the only other study of this nature, on Caucasoids, was $3.1 \pm 0.5 \%$ (Rosenblum et al. 1984). This is significantly less than the amount 
of variation observed in 2-D PAGE preparations of plasma by ourselves (Asakawa et al. 1984, 1985; Neel et al. 1984; Rosenblum et al. $1983 a, b)$ but significantly more than that observed by others examining a variety of other types of preparations (Comings, 1982; McConkey et al. 1979; Smith et al. 1980; Walton et al. 1979; but see Goldman \& Merril, 1983). We have previously pointed out that there is an inverse relationship between the number of polypeptides scored by the investigator and the index of heterozygosity reported, and suggested that efforts to score more polypeptides than the preparations warrant, especially in the absence of family material, is a major reason for the lower indices reported by many other investigators (Neel et al. 1985).

On the other hand, there may indeed be differences between tissues, cell types, and plasma in the frequency with which genetic variants are encountered. The index of heterozygosity of $6 \cdot 1 \%$ from 1-DE is dominated by plasma and erythrocyte lysate proteins. In Japanese our index from 2-D PAGE for plasma and lysate preparations combined is $4 \cdot 3 \pm 0 \cdot 2 \%$. We have not yet examined some of the human tissues (brain, kidney) and cell types (lymphocytes, fibroblasts) examined by others, and would not exclude the possibility of some real differences, albeit, we suspect, not as great at these earlier reports suggest.

For 7 of the 14 polypeptides exhibiting genetic variation in this study, coordinate variation was observed in a second (or third) nearby polypeptide; presumably one of the two (or three) polypeptides is primary and the other(s) derivative. Two of the variant polypeptides are in the MW axis. These findings correspond to our previous experience, that about $20 \%$ of polypeptide variants in 2-D gels are primarily in the MW axis (Asakawa et al. 1985).

Of the 8 presumably identical polypeptides that were variable either in these gels or the gels from Caucasoids scored in Ann Arbor, C-012 and D-018 were polymorphic in Japanese but not in Caucasoids, and there were significant allele frequency differences for C-022 (GLO1) and D-024. If the present data are combined with the data on plasma proteins, ethnically significant differences in allele frequencies have been observed in 4 of the 10 polymorphic systems which have been identified in $2-\mathrm{D}$ gels in both populations. These differences are based on small numbers, and some may not persist, but it seems clear these systems will be of great anthropological interest.

In our opinion, the gels now have the reproducibility to allow an appropriately chosen battery of polypeptides to be used for studies of the rate with which mutation results in electromorphs, an application of the 2-D PAGE technique, which, of course, demands a greater degree of technical reliability than studies of genetic variation. However, further improvements are in sight. For those preparations which cannot be isotopically labelled, the chief possibility would appear to be the use of multiple $\mathrm{pH}$ gradients, as a means of achieving not only better separation of spots but also better visualization of some of the polypeptides at the current upper and lower isoelectric focusing bounds. For samples that can be labelled isotopically, such as cultured lymphocytes or fibroblasts, the technique of electronic autofluorography to impart a greater dynamic range to quantification of polypeptide spots (Davidson \& Case, 1982) should be especially useful in the search for null variants characterized by lack of polypeptide formation.

There is an urgent need for standardization of procedures between laboratories. Without this, appropriate comparisons are impossible. For instance, as a result of the relatively minor technical differences between the procedures used in the present study and in the University of Michigan study, only 27 of the 46 polypeptides scored in the Michigan study could be 
unequivocally identified by ourselves. In time, as the various polypeptides are specifically identified, this problem will decrease. For the present, with the techniques evolving so rapidly, it is probably premature to attempt a standardization.

There is currently considerable interest in the possibility that the study of mutation can be carried to the DNA level (World Health Organization, 1985; Delehanty et al. 1986). This is an exciting prospect. We suggest that in the context of population monitoring, the experience with 2-D PAGE can provide an important standard of comparison. Perhaps the most appropriate way to proceed is in terms of output per technician year. In such a calculation there are two considerations. Firstly, a certain fraction of gels will for various reasons be substandard and have to be repeated; we estimate about $10 \%$ or less. Secondly, up to this point in time we have routinely run preparations from both parents together with the individual's preparation, to have an immediate check available on the genetic nature of any apparent variant. Our familiarity with the lysate patterns has now reached the point where we feel parental gels need not be run routinely, but only in the event of an unusual finding, i.e. a rare variant rather than a common polymorphism. Let us estimate that, for the present, an examination of the corresponding gels from the parents will be necessary for $30 \%$ of the primary gels. Thus of each 100 gels, roughly 56 will be primary gels, 34 parental gels, and 10 repeats. In our experience, a team of two technicians should, with allowance for re-runs, family studies, scoring, etc., completely process the equivalent of at least 25 test gels per week, each gel yielding information on some 50 polypeptides. This amounts to some 125000 locus tests per year for mutations resulting in electromorphs. Let us assume that electrophoresis detects $1 / 3$ of the nucleotide substitutions in exons and that the average polypeptide requires 1,200 nucleotides for its specification. Then the number of nucleotides effectively scanned per year by two technicians is $(1200 / 3) \times 125000=50000000$, at a relatively low cost for supplies. The $2-\mathrm{D}$ methodology should thus be relatively effective as compared with current DNA technologies (see Delehanty et al. 1986) in screening for mutations resulting in nucleotide substitutions. This would recommend the 2-D technique for studies of chemical mutagenesis, where nucleotide substitutions appear to be a major type of lesion. On the other hand, a principal current drawback of the 2-D approach to the study of mutation is the inability to detect heterozygotes for gene-inactivation mutations with the necessary accuracy, and the technique would not be as powerful, relatively speaking, in studies of radiation mutagenesis, where loss-of-activity mutations appear to predominate.

Note added in proof: In the text we accepted on the basis of position the identity of our C-022 with the RBC1 of Goldman et al. (1985), which they had identified as glyoxalase 1 (GLO1). We have now determined the phenotypes for GLO1 of the 51 individuals who constitute the subjects of this paper. These were GLO1, 1; GLO1 1-2, 12; and GLO1 2, 38. In every case, the typings for GLO1 coincided with the typings for our spot C-022.

The Radiation Effects Research Foundation (formerly ABCC) was established in April 1975 as a private non-profit-making Japanese foundation, supported equally by the Government of Japan through the Ministry of Health and Welfare, and the Government of the United States through the National Academy of Sciences under contract with the Department of Energy. We are grateful to Mrs E. Nishikori and Mr S. Kaneoka for their technical assistance on 2-D gel electrophoresis. 


\section{REFERENCES}

Asakawa, J., Takahashi, N., Rosenblum, B. B. \& Negl, J. V. (1984). Analysis of genetic variation in Amerindian sera by 2-D PAGE. In Electrophoresis ' 83 (ed. H. Hirai), p. 189. Berlin, New York: Walter de Gruyter.

Gruyter.
asakawa, J., Takahashi, N., Rosenblum, B. B. \& Neel, J. V. (1985). Two-dimensional gel studies of genetic variation in the plasma proteins of Amerindians and Japanese Hum. Genet. 70, 220-230.

Comings, D. E. (1982). Two-dimensional gel electrophoresis of human brain proteins. III. Genetic and non-genetic variations in 145 brains. Clin. Chem. 28, 798-804.

Davidson, J. B. \& CASE, A. L. (1982). Rapid electronic autofluorography of labeled macromolecules on two-dimensional gels. Science 215, 1398-1400.

Delehanty, J., White, R. L. \& Mendelsohn, M. L. (1986). Approaches to determining mutation rates in human DNA: ICPEMC Meeting Report No. 1, Mut. Res. 167, 215-232.

Goldman, D., Goldin, L. R., Rathnagiri, P., O'Brien, S. J., Egeland, J. A. \& Merril, C. R. (1985). Twenty-seven protein polymorphisms by two-dimensional electrophoresis of serum, erythrocytes, and fibroblasts in two pedigrees. Am. J. Hum. Genet. 37, 898-911.

Goldman, D. \& MerriL, C. R. (1983). Human lymphocyte polymorphisms detected by quantitative twodimensional electrophoresis. Am. J. Hum. Genet. 35, 827-837.

Harris, H. (1980). The Principles of Human Biochemical Genetics. 3rd Edition. Amsterdam: Elsevier/North-Holland.

Kondo, I., Yamamoto, T., Yamakawa, K., Harada, S., Oishi, H., Nishigaki, I. \& Hamaguchi, H. (1984a). Genetic analysis of human lymphocyte proteins by two-dimensional gel electrophoresis. VI. Identification of esterase $\mathrm{D}$ in the two-dimensional gel electrophoresis pattern of cellular proteins. Hum. Genet. 66, 248-251.

Kondo, I., Nishigaki, I., Yamakawa, K. \& Hamaguchi, H. (1984b). The esterase D polymorphism: analysis of esterase D 7 by two-dimensional gel electrophoresis. Jpn. J. Hum. Genet. 29, 27-30.

McConkey, E. H., Taylor, B. J. \& Phan, D. (1979). Human heterozygosity: a new estimate. Proc. Natl. Acad. Sci. USA 76, 6500-6504.

Muler, O.J., Drayna, D. \& Goodfellow, P. (1984). Report of the committee on the genetic constitution of the X and Y chromosomes. Cytogenet. Cell Genet. 37, 176-204.

Neel, J. V., Bater, L., Hanash, S. M. \& Erickson, R. P. (1985). The frequency of polymorphisms for alleles encoding for proteins of the liver of domesticated mice. J. Hered. 76, $314-320$.

Neel, J. V., Rosenblum, B. B., Sing, C. F., Skolnick, M. M., Hanash, S. M. \& Sternberg, S. (1984). Adapting two-dimensional gel electrophoresis to the study of human germ-line mutation rates. In Methods and Applications of Two-Dimensional Gel Electrophoresis of Proteins (ed. J. E. Celis and R. Bravo), p. 259. New York: Academic Press.

Neel, J. V. Satoh, C., Hamilton, H. B., Otake, M., Goriki, K., Kageoka, T., Fujita, M., Neriishi, S. \& AsAKAWA, J. (1980). Search for mutations affecting protein structure in children of atomic bomb survivors: Preliminary report. Proc. Natl. Acad. Sci. USA 77, 4221-4225.

Rosenblum, B. B., Hanash, S. M. \& Neel, J. V. (1983b). High-resolution separation of plasma proteins, applications to genetic analysis. In Biologie Prospective, $5^{e}$ Colloque International de Pont-à-Mousson (ed. M.-M. Galteau, G. Siest and J. Henny), p. 85. Paris: Masson.

Rosenblum, B. B., Neel, J. V. \& Hanash, S. M. (1983a). Two-dimensional electrophoresis of plasma polypeptides reveals 'high' heterozygosity indices. Proc. Natl. Acad. Sci. USA 80, 5002-5006.

Rosenblum, B. B., Neel, J. V., Hanash, S. M., Joseph, J. L. \& Yew, N. (1984). Identification of genetic variants in erythrocyte lysate by two-dimensional gel electrophoresis. Am. J. Hum. Genet. 36, 601-612.

Satoh, C., Awa, A. A., Neel, J. V., Schull, W. J., Kato, H., Hamilton, H. B., Otake, M. \& Goriki, K. (1982). Genetic effects of atomic bombs. In Human Genetics, Part A: The Unfolding Genome (ed. B. BonnéTamir, T. Cohen and R. M. Goodman), p. 267. New York: Alan R. Liss.

Satoh, C., Neel, J. V., Miura, A., Ueno, C., Arakawa, H., Omine, H., Goriki, K. \& Fujita, M. (1985). Inherited thermostability variants of seven enzymes in a Japanese population. Ann. Hum. Genet. 49, 11-22.

Satoh, C., Neel, J. V., Yamashita, A., Goriki, K., Fujita, M. \& Hamilton, H. B. (1983). The frequency among Japanese of heterozygotes for deficiency variants of 11 enzymes. Am. J. Hum. Genet. 35, 656-674.

Smith, S. C., Racine, R. R. \& Lanaley, C. H. (1980). Lack of genic variation in the abundant proteins of human kidney. Genetics 96, 967-974.

Walton, K. E., Styer, D. \& Gruenstein, E. I. (1979). Genetic polymorphism in normal human fibroblasts as analyzed by two-dimensional polyacrylamide gel electrophoresis. J. Biol. Chem. 254, 7951-7960.

World Health Organization (1985). Report of WhO Task Group on Prevention of Avoidable Mutational Disease (HMG/TG/84.3). WHO: Geneva.

Wray, W., Boulikas, T., Wray, V. P. \& Hancock, R. (1981). Silver staining of proteins in polyacrylamide gels. Anal. Biochem. 118, 197-203. 